\title{
The efficacy of BCG TICE and BCG Connaught in a cohort of 2,099 patients with T1G3 non-muscle-invasive bladder cancer
}

J. Alfred Witjes, M.D., Ph.D. ${ }^{\mathrm{a}, *}$, Guido Dalbagni, M.D. ${ }^{\mathrm{b}}$, Robert J. Karnes, M.D. ${ }^{\mathrm{c}}$, Shahrokh Shariat, M.D. ${ }^{\mathrm{d}}$, Steven Joniau, M.D., Ph.D. ${ }^{\mathrm{e}}$, Joan Palou, M.D., Ph.D. ${ }^{\mathrm{f}}$,

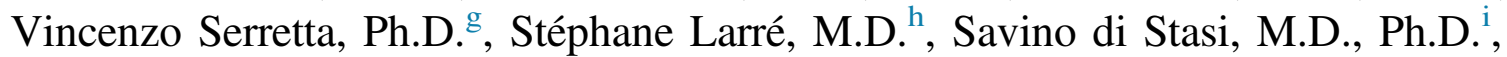
Renzo Colombo, M.D., Ph.D. ${ }^{J}$, Marek Babjuk, M.D., C.Sc. ${ }^{k}$, Per-Uno Malmström, M.D. , Nuria Malats, M.D. ${ }^{\mathrm{m}}$, Jacques Irani, Ph.D. ${ }^{\mathrm{n}}$, Jack Baniel, Ph.D. ${ }^{\circ}$, Tommaso Cai, M.D. ${ }^{\mathrm{p}}$, Eugene Cha, M.D. ${ }^{\mathrm{q}, \mathrm{b}}$, Peter Ardelt, M.D. ${ }^{\mathrm{r}}$, John Varkarakis, Ph.D. ${ }^{\mathrm{s}}$, Riccardo Bartoletti, M.D. Martin Spahn, M.D., Ph.D. ${ }^{u}$, Francesca Pisano, M.D. ${ }^{\mathrm{v}}$, Paolo Gontero, M.D. ${ }^{\mathrm{v}}$, Richard Sylvester, M.D. ${ }^{\mathrm{w}}$

\author{
${ }^{a}$ Department of Urology, RadboudUMC, Nijmegen, The Netherlands \\ ${ }^{\mathrm{b}}$ Urology Service, Department of Surgery, Memorial Sloan-Kettering Cancer Center, New York, NY \\ ${ }^{\mathrm{c}}$ Department of Urology, Mayo Clinic, Rochester, MN \\ ${ }^{\mathrm{d}}$ Department of Urology, Comprehensive Cancer Center Medical University Vienna, Vienna, Austria \\ ${ }^{\mathrm{e}}$ Oncologic and Reconstructive Urology, Department of Urology, University Hospitals Leuven, Leuven, Belgium \\ ${ }_{\mathrm{f}}^{\mathrm{f}}$ Department of Urology, Fundacio Puigvert, University of Barcelona, Barcelona, Spain \\ ${ }^{\mathrm{g}}$ Department of Urology, Paolo Giaccone General Hospital, Palermo, Italy \\ ${ }^{\mathrm{h}}$ Department of Surgical Science, John Radcliffe Hospital, University of Oxford, Oxford, UK

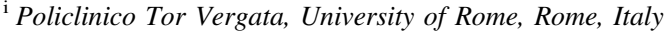 \\ ${ }^{j}$ Dipartimento di Urologia, Universita` Vita-Salute, Ospedale S. Raffaele, Milan, Italy \\ ${ }^{\mathrm{k}}$ Department of Urology, Motol Hospital, 2nd Faculty of Medicine, Charles, University of Prague, Prague, Czech Republic \\ ${ }^{1}$ Department of Surgical Sciences, Uppsala University, Uppsala, Sweden \\ ${ }^{\mathrm{m}}$ Department of Urology, Spanish National Cancer Research Centre-Madrid, Madrid, Spain \\ ${ }^{\mathrm{n}}$ Department of Urology, Centre Hospitalier Universitaire La Milétrie, University of Poitiers, Poitiers, France \\ ${ }^{\circ}$ Institute of Urology, Rabin Medical Center, Petach Tikva and Sackler Faculty of Medicine, Tel Aviv University, Tel Aviv, Israel \\ ${ }^{\mathrm{p}}$ Department of Urology, Santa Chiara Hospital, Trento, Italy \\ ${ }^{\mathrm{q}}$ Department of Urology, Weill Medical College of Cornell University, New York, NY \\ ${ }^{\mathrm{r}}$ Facharzt fur Urologie, Abteilung fur Urologie, Chirurgische Universitatsklinik, Freiburg, Germany \\ ${ }^{\mathrm{s}}$ Department of Urology, Sismanoglio Hospital, University of Athens, Athens, Greece \\ ${ }^{\mathrm{t}}$ Urology Unit, S. Maria Annunziata Hospital, University of Florence, Florence, Italy \\ u Department of Urology, University Hospital of Wuerzburg, Wuertzburg, Germany \\ ${ }^{v}$ Department of Surgical Sciences, Molinette Hospital, University of Studies of Turin, Turin, Italy \\ ${ }^{\mathrm{w}}$ Formerly Department of Biostatistics, EORTC Headquarters, Brussels, Belgium
}

Received 27 January 2016; received in revised form 25 May 2016; accepted 30 May 2016

\begin{abstract}
Background: Potential differences in efficacy of different bacillus Calmette-Guérin (BCG) strains are of importance for daily practice, especially in the era of BCG shortage.

Objective: To retrospectively compare the outcome with BCG Connaught and BCG TICE in a large study cohort of pT1 high-grade non-muscle-invasive bladder cancer patients.
\end{abstract}

\footnotetext{
${ }^{*}$ Corresponding author. Tel.: +00-31-24-3613735.

E-mail addresses: fred.witjes@radboudumc.nl, francescapisano85@gmail.com (J.A. Witjes).
} 
Design, setting, and participants: Individual patient data were collected for 2,451 patients with primary T1G3 tumors from 23 centers who were treated with BCG for the first time between 1990 and 2011.

Outcome measurements and statistical analysis: Using Cox multivariable regression and adjusting for the most important prognostic factors in this nonrandomized comparison, BCG Connaught and TICE were compared for time to recurrence, progression, and the duration of cancer specific survival and overall survival.

Results and limitations: Information on the BCG strain was available for 2,099 patients: 957 on Connaught and 1,142 on TICE. Overall, $765(36 \%)$ patients received some form of maintenance BCG, 560 (59\%) on Connaught and 205 (18\%) on TICE. Without maintenance, Connaught was more effective than TICE only for the time to first recurrence (hazard ratio $[\mathrm{HR}]=1.48 ; 95 \% \mathrm{CI}: 1.20-1.82 ; P<0.001$ ). With maintenance, TICE was more effective than Connaught for the time to first recurrence $(\mathrm{HR}=0.66 ; 95 \%$ CI: $0.47-0.93 ; P=0.019)$ with a trend for cancer specific survival $(\mathrm{HR}=0.36 ; 95 \% \mathrm{CI}: 0.14-0.92 ; P=0.033)$. For time to progression and overall survival, Connaught and TICE had a similar efficacy. Compared to no maintenance therapy, maintenance BCG significantly reduced the risk of recurrence, progression and death, both overall, and disease specific, for TICE, but not for Connaught.

Conclusions: We found that BCG Connaught results in a lower recurrence rate as compared with BCG TICE when no maintenance is used. However, the opposite is true when maintenance is given.

Patient summary: As there is currently a BCG shortage, information on the efficacy of different BCG strains is important. In this nonrandomized retrospective comparison in over 2,000 patients, we found that BCG Connaught reduces the recurrence rate compared to BCG TICE when no maintenance is used, but the opposite is true when maintenance is given. (C) 2016 Elsevier Inc. All rights reserved.

Keywords: T1G3; bladder cancer; high grade; progression; recurrence; BCG Connaught; BCG Tice; BCG vaccine; Immunotherapy; Intravesical drug administration

\section{Introduction}

Bacillus Calmette-Guérin (BCG) remains an important treatment option after transurethral resection (TUR) of bladder tumor in patients with non-muscle-invasive bladder cancer (NMIBC). It is recommended in intermediate and high-risk patients, although intravesical chemotherapy also has a role in intermediate-risk patients [1].

Several different BCG strains have been used for treating NMIBC, all originating from the original vaccine for tuberculosis. They have been distributed across countries and continents since many decades, resulting in an accumulation of mutations [2]. Whether these differences have resulted in differences in efficacy or toxicity for either tuberculosis vaccination or NMIBC treatment remains largely unknown [2].

In NMIBC, the current BCG shortage has made the comparison of different strains and a strain's potential replacement by another strain particularly important. There are, however, only few randomized controlled trial (RCT) comparing different BCG strains. The 2 largest are a 1995 Dutch trial and a 2014 Swiss trial. The Dutch study showed that an induction course of BCG RIVM reduced recurrences compared with an induction course of BCG TICE [3]. The Swiss trial [2] found an induction course of BCG Connaught to be significantly better in reducing recurrences than an induction course of BCG TICE. These authors suggested that the superiority of BCG Connaught was because of a superior immunogenicity, as demonstrated in mice, and genetic differences. A recent Japanese trial compared BCG Tokyo172 and BCG Connaught in 129 patients with NMIBC [4]. Complete response (CR) rates in (concomitant) carcinoma in situ (CIS) and 2-year recurrence-free survival (RFS) were similar in both arms.
In our study, a large European cohort of patients with high-risk NMIBC, we have retrospectively compared the 2 most widely used BCG strains, Connaught and TICE, with respect to recurrence, progression, cancer specific survival, and overall survival (OS).

\section{Patients and methods}

\subsection{Database}

Patients, methods, and results have been previously reported [5]. Individual patient data were retrospectively collected for patients with high-grade/G3 T1 urothelial carcinoma who received at least an induction course of BCG that started between 1990 and 2011. Patients with a previous NMIBC that was not $\mathrm{T} 1$ high grade/G3 were eligible if they did not receive BCG for that tumor. In addition to patient and tumor characteristics, information on BCG strain, dose, total number of instillations, and reasons for stopping BCG were recorded. Instillations beyond 6 were defined as maintenance BCG. End points were time to first recurrence (any stage or grade), progression to muscleinvasive disease, duration of cancer specific survival, and OS.

\subsection{Statistical analysis}

As the 2 strains were not assigned by randomization and there are imbalances in the distribution of the prognostic factors, Cox proportional hazards multivariable regression analyses were performed to adjust for the number of the adverse prognostic factors using the most important variables that had been previously identified: age $(<70 \mathrm{y}$, $\geq 70 \mathrm{y}$ ) for progression and survival, number of tumors 
(single and multiple) for recurrence, tumor size $(<3 \mathrm{~cm}$, $\geq 3 \mathrm{~cm}$ ) for recurrence, progression and survival, and concomitant CIS (no and yes) for progression [5]. A second confirmatory analysis which included each of these variables in the models instead of the number of adverse factors provided similar results. Adjustment for the use of maintenance, tests for interaction of BCG strain and maintenance, and separate analyses in patients receiving and not receiving maintenance were done.

Times to events were calculated taking the date of starting BCG as time zero. OS was estimated using the Kaplan-Meier technique. To take into account patients who died before the event of interest (competing risk), times to the other events were estimated using cumulative incidence functions. Patients without an event were censored at the last date of follow-up.

\section{Results}

In this cohort of 2,451 patients with primary $\mathrm{T} 1 \mathrm{G} 3$, information on treatment with Connaught or TICE was available in 18 of 23 centers with 2,099 patients: 957 on Connaught and 1,142 on TICE. Maintenance BCG was given in 765 patients $(36 \%), 560$ of 957 patients $(59 \%)$ on Connaught, and 205 of 1,142 patients $(18 \%)$ on TICE. The distribution of patient and tumor characteristics is given in Table 1. More patients on TICE had multiple tumors, large tumors, restaging TURs, and 1 or more adverse risk factors for survival. Fewer TICE patients received maintenance.

Median follow-up was 5.2 year with a maximum followup of 18.7 year. The number of patients who recurred, progressed and died is summarized in Tables 2-4.

\subsection{Time to first recurrence}

Overall, 439 patients (46\%) on Connaught and 625 (55\%) on TICE recurred, $50 \%$ on Connaught and $59 \%$ on TICE without maintenance, $43 \%$ and $34 \%$ with maintenance, respectively (Tables 2-4).

After adjustment for the risk of recurrence, maintenance BCG prolonged the time to first recurrence (hazard ratio $[\mathrm{HR}]=0.60 ; 95 \% \mathrm{CI}: 0.51-0.70 ; P<0.001)$. The difference was mainly found in patients receiving TICE $(\mathrm{HR}=$ 0.37 ; $95 \%$ CI: $0.26-0.51 ; P<0.001)$ rather than in patients receiving Connaught $(\mathrm{HR}=0.85 ; 95 \% \mathrm{CI}: 0.691 .06 ; P$ $=0.15)$.

The difference in time to first recurrence between strains, after adjustment for the risk of recurrence and use of maintenance, was significant $(\mathrm{HR}=1.18 ; 95 \% \mathrm{CI}: 1.00$ 1.39; $P=0.047$ ) in favor of Connaught. However, the test for interaction between strain and maintenance BCG was significant $(P<0.001)$. Adjusting for the risk of recurrence, there was a significantly longer time to first recurrence on Connaught as compared with TICE when no maintenance was given $(\mathrm{HR}=1.48 ; 95 \% \mathrm{CI}: 1.201 .82$;
Table 1

Patient and tumor characteristics

\begin{tabular}{|c|c|c|c|}
\hline & $\begin{array}{l}\text { BCG Connaught } \\
(n=957)\end{array}$ & $\begin{array}{l}\text { BCG TICE } \\
(n=1,142)\end{array}$ & $\begin{array}{l}\text { Total } \\
(n=2,099)\end{array}$ \\
\hline \multicolumn{4}{|l|}{ Age } \\
\hline$<70$ & $556(58.1 \%)$ & $638(55.9 \%)$ & $1,194(56.9 \%)$ \\
\hline$\geq 70$ & $401(41.9 \%)$ & $504(44.1 \%)$ & $905(43.1 \%)$ \\
\hline \multicolumn{4}{|l|}{ Sex } \\
\hline Male & $792(82.8 \%)$ & $928(81.3 \%)$ & $1,720(81.9 \%)$ \\
\hline Female & $165(17.2 \%)$ & $214(18.7 \%)$ & $379(18.1 \%)$ \\
\hline \multicolumn{4}{|l|}{ Tumor status } \\
\hline Primary & $835(87.3 \%)$ & $1,024(89.7 \%)$ & $1,859(88.6 \%)$ \\
\hline Recurrent & $122(12.8 \%)$ & $118(10.3 \%)$ & $240(11.4 \%)$ \\
\hline \multicolumn{4}{|c|}{ Previous intravesical chemotherapy } \\
\hline No & $913(95.4 \%)$ & $1,066(93.4 \%)$ & $1,979(94.3 \%)$ \\
\hline Yes & $44(4.6 \%)$ & $76(6.6 \%)$ & $120(5.7 \%)$ \\
\hline \multicolumn{4}{|c|}{ Number of tumors } \\
\hline Single & $381(45.5 \%)$ & $420(36.9 \%)$ & $801(40.5 \%)$ \\
\hline Multiple & $457(54.5 \%)$ & $719(63.1 \%)$ & $1,176(59.5 \%)$ \\
\hline Missing & 119 & 3 & 122 \\
\hline \multicolumn{4}{|c|}{ Largest tumor size } \\
\hline$<3 \mathrm{~cm}$ & $557(75.0 \%)$ & $440(70.3 \%)$ & $997(72.8 \%)$ \\
\hline$\geq 3 \mathrm{~cm}$ & $186(25.0 \%)$ & $186(29.7 \%)$ & $372(27.2 \%)$ \\
\hline Missing & 214 & 516 & 730 \\
\hline \multicolumn{4}{|c|}{ Concomitant CIS } \\
\hline No & $731(76.4 \%)$ & $841(73.6 \%)$ & $1,572(74.9 \%)$ \\
\hline Yes & $226(23.6 \%)$ & $301(26.4 \%)$ & $527(25.1 \%)$ \\
\hline \multicolumn{4}{|c|}{ Restaging TUR before } \\
\hline \multicolumn{4}{|l|}{$\mathrm{BCG}$} \\
\hline No & $582(60.8 \%)$ & $482(42.2 \%)$ & $1,064(50.7 \%)$ \\
\hline Yes & $303(31.7 \%)$ & $558(48.9 \%)$ & $861(41.0 \%)$ \\
\hline Missing & $72(7.5 \%)$ & $102(8.9 \%)$ & $174(8.3 \%)$ \\
\hline \multicolumn{4}{|c|}{ Risk factors for recurrence } \\
\hline 0 & $278(37.5 \%)$ & $260(41.5 \%)$ & $538(39.3 \%)$ \\
\hline 1 & $347(46.8 \%)$ & $284(45.4 \%)$ & $631(46.1 \%)$ \\
\hline 2 & $117(15.8 \%)$ & $82(13.1 \%)$ & $199(14.6 \%)$ \\
\hline Missing & 215 & 516 & 731 \\
\hline \multicolumn{4}{|c|}{ Risk factors for progression } \\
\hline 0 & $234(31.5 \%)$ & $162(25.9 \%)$ & $396(28.9 \%)$ \\
\hline 1 & $340(45.8 \%)$ & $294(47.0 \%)$ & $634(46.3 \%)$ \\
\hline 2 & $142(19.1 \%)$ & $136(21.7 \%)$ & $278(20.3 \%)$ \\
\hline 3 & $27(3.6 \%)$ & $34(5.4 \%)$ & $61(4.5 \%)$ \\
\hline Missing & 214 & 516 & 730 \\
\hline \multicolumn{4}{|c|}{ Risk factors for survival } \\
\hline 0 & $332(44.7 \%)$ & $212(33.9 \%)$ & $544(39.7 \%)$ \\
\hline 1 & $324(43.6 \%)$ & $319(51.0 \%)$ & $643(47.0 \%)$ \\
\hline 2 & $87(11.7 \%)$ & $95(15.2 \%)$ & $182(13.3 \%)$ \\
\hline Missing & 214 & 516 & 730 \\
\hline \multicolumn{4}{|l|}{ Maintenance } \\
\hline No & $397(41.5 \%)$ & $937(82.1 \%)$ & $1,334(63.6 \%)$ \\
\hline Yes & $560(58.5 \%)$ & $205(17.9 \%)$ & $765(36.4 \%)$ \\
\hline
\end{tabular}

$P<0.001$ ), whereas with maintenance there was a significantly longer time to first recurrence on TICE $(\mathrm{HR}=0.66$; 95\% CI: 0.47 0.93; $P=0.019$ ) (Tables 3 and 4 and Fig. 1). 
Table 2

Multivariable adjusted comparison of BCG Connaught to BCG TICE: all patients

\begin{tabular}{|c|c|c|c|c|c|}
\hline & $\begin{array}{l}\text { BCG Connaught } \\
(n=957) N,(\%)\end{array}$ & $\begin{array}{l}\text { BCG TICE } \\
(n=1,142) N,(\%)\end{array}$ & $\begin{array}{l}\text { Hazard ratio } \\
(95 \% \mathrm{CI})\end{array}$ & $P$ value & $\begin{array}{l}P \text { value Interaction } \\
\text { with Maintenance }\end{array}$ \\
\hline \multicolumn{6}{|c|}{ Recurrence } \\
\hline No & $518(54.1)$ & $517(45.3)$ & $1.18(1.00-1.39)$ & 0.047 & $<0.001$ \\
\hline Yes & 439 (45.9) & $625(54.7)$ & & & \\
\hline \multicolumn{6}{|c|}{ Progression } \\
\hline No & 779 (81.4) & $940(82.3)$ & $1.08(0.84-1.39)$ & 0.55 & 0.16 \\
\hline Yes & $178(18.6)$ & $202(17.7)$ & & & \\
\hline \multicolumn{6}{|l|}{ Survival } \\
\hline Alive & $713(74.5)$ & $868(76.0)$ & $1.02(0.81-1.28)$ & 0.87 & 0.13 \\
\hline Dead & $244(25.5)$ & $274(24.0)$ & & & \\
\hline \multicolumn{6}{|c|}{ Death bladder cancer } \\
\hline No & $864(90.3)$ & $1,054(92.3)$ & $0.73(0.50-1.06)$ & 0.10 & 0.12 \\
\hline Yes & $93(9.7)$ & $88(7.7)$ & & & \\
\hline
\end{tabular}

HR $>1$ favors Connaught, HR $<1$ favors TICE.

\subsection{Time to progression}

Overall, 178 patients (19\%) on Connaught and 202 (18\%) on TICE progressed, $21 \%$ on Connaught, and $18 \%$ on TICE without maintenance, $17 \%$ and $14 \%$ with maintenance, respectively (Tables 2-4).

After adjustment for the risk of progression, maintenance BCG prolonged the time to progression for TICE $(\mathrm{HR}=$ 0.59; 95\% CI: 0.36-0.94; $P=0.03$ ), but not for Connaught patients $(\mathrm{HR}=0.94 ; 95 \% \mathrm{CI}: 0.67-1.31 ; P=0.71)$.

When adjusted for the risk of progression (age $\geq 70 \mathrm{y}$, size $\geq 3 \mathrm{~cm}$, CIS) and use of maintenance, there was not a significant difference in the time to progression between the strains $(\mathrm{HR}=1.08 ; 95 \% \mathrm{CI}: 0.84-1.39 ; P=0.55)$ (Table 2 and Fig. 2). The test for interaction between strain and maintenance BCG was not significant $(P=0.16)$.

Table 3

Multivariable adjusted comparison of BCG Connaught to BCG TICE: patients without BCG maintenance

\begin{tabular}{|c|c|c|c|c|}
\hline & $\begin{array}{l}\text { BCG Connaught } \\
(n=397) N, \\
(\%)\end{array}$ & $\begin{array}{l}\text { BCG TICE } \\
(n=937) N, \\
(\%)\end{array}$ & $\begin{array}{l}\text { Hazard ratio } \\
(95 \% \text { CI) }\end{array}$ & $P$ value \\
\hline \multicolumn{5}{|c|}{ Recurrence } \\
\hline No & $199(50.1)$ & $381(40.7)$ & $1.48(1.20-1.82)$ & $<0.001$ \\
\hline Yes & $198(49.9)$ & $556(59.3)$ & & \\
\hline \multicolumn{5}{|c|}{ Progression } \\
\hline No & $313(78.8)$ & $764(81.5)$ & $1.21(0.88-1.65)$ & 0.24 \\
\hline Yes & $84(21.2)$ & $173(18.5)$ & & \\
\hline \multicolumn{5}{|l|}{ Survival } \\
\hline Alive & $290(73.1)$ & $701(74.8)$ & $1.18(0.89-1.56)$ & 0.25 \\
\hline Dead & 107 (26.9) & $236(25.2)$ & & \\
\hline \multicolumn{5}{|c|}{ Death bladder cancer } \\
\hline No & 355 (89.4) & $855(91.2)$ & $0.90(0.57-1.40)$ & 0.63 \\
\hline Yes & $42(10.6)$ & $82(8.8)$ & & \\
\hline
\end{tabular}

HR $>1$ favors Connaught, HR $<1$ favors TICE.

\subsection{Duration of Survival}

Overall, 244 patients $(26 \%)$ on Connaught and 274 (24\%) on TICE died due to any cause, $27 \%$ on Connaught and $25 \%$ on TICE without maintenance, $24 \%$ and $18 \%$ with maintenance, respectively.

After adjustment for the risk of death, maintenance BCG significantly prolonged the duration of survival in TICE patients $(\mathrm{HR}=0.63 ; 95 \% \mathrm{CI}: 0.41-0.97 ; P=0.04)$, but not in Connaught patients (HR $=0.94 ; 95 \%$ CI: 0.70-1.25; $P=0.65)$.

There was not a significant difference in survival between strains (Table 2 and Fig. 3) after adjusting for the risk of death and use of maintenance (HR $=1.02 ; 95 \%$ CI: $0.81-1.28 ; P=0.87)$. The test for interaction between strain and maintenance was not significant $(P=0.13)$.

Table 4

Multivariable adjusted comparison of BCG Connaught to BCG TICE: patients with BCG maintenance

\begin{tabular}{llrll}
\hline & $\begin{array}{l}\text { BCG Connaught } \\
(n=560) N, \\
(\%)\end{array}$ & $\begin{array}{l}\text { BCG TICE } \\
(n=205) \\
N,(\%)\end{array}$ & $\begin{array}{l}\text { Hazard ratio } \\
(95 \% \text { CI })\end{array}$ & $P$ value \\
& & & \\
\hline Recurrence & & & & \\
$\quad$ No & $319(57.0)$ & $136(66.3)$ & $0.66(0.47-0.93)$ & 0.019 \\
Yes & $241(43.0)$ & $69(33.7)$ & & \\
Progression & & & & \\
$\quad$ No & $466(83.2)$ & $176(85.9)$ & $0.79(0.49-1.29)$ & 0.35 \\
Yes & $94(16.8)$ & $29(14.1)$ & & \\
Survival & & $167(81.5)$ & $0.72(0.46-1.12)$ & 0.14 \\
$\quad$ Alive & $423(75.5)$ & $38(18.5)$ & & \\
Dead & $137(24.5)$ & & & \\
Death bladder & & & & \\
$\quad$ cancer & & & & \\
$\quad$ No & $509(89.9)$ & $199(97.1)$ & $0.36(0.14-0.92)$ & 0.033 \\
Yes & $51(9.1)$ & $6(2.9)$ & & \\
\hline
\end{tabular}

HR $>1$ favors Connaught, HR $<1$ favors TICE. 


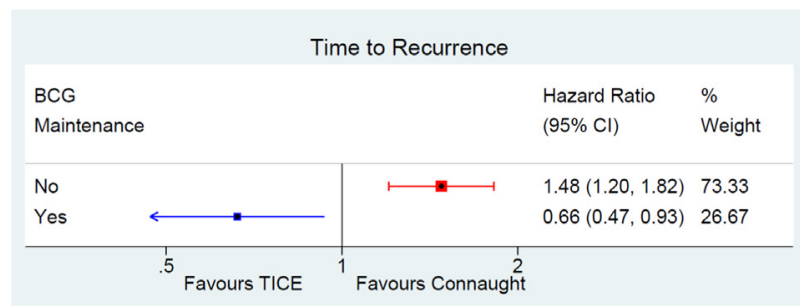

Fig. 1. Forest plot time to first recurrence. (Color version of figure is available online.)

\subsection{Time to death due to bladder cancer}

Overall, 93 patients $(10 \%)$ on Connaught and $88(8 \%)$ on TICE died because of bladder cancer, $11 \%$ on Connaught and $9 \%$ on TICE without maintenance, $9 \%$ and $3 \%$ with maintenance, respectively.

After adjustment for the risk of death, maintenance BCG prolonged the time to death due to bladder cancer in patients treated with TICE $(\mathrm{HR}=0.37$; 95\% CI: $0.15-0.93 ; P=$ $0.034)$, but not in patients treated with Connaught $(\mathrm{HR}=0.79$; 95\% CI: $0.50-1.23 ; P=0.30$ ).

There was no significant difference in the time to death due to bladder cancer according to strain when adjusted for the risk of death and the use of maintenance $(\mathrm{HR}=0.73 ; 95 \% \mathrm{CI}: 0.50-1.06 ; P=0.10)$. The test for interaction between strain and maintenance BCG was not statistically significant $(P=0.12)$; however, when maintenance was used, there was a suggestion of a significantly longer time to death due to bladder cancer with TICE (Table 4 and Fig. 4).

\section{Discussion}

The current dataset remains the largest ever reported on patients with pT1G3 bladder tumors who received BCG as primary treatment.

Our results showed that without maintenance therapy, Connaught was more effective than TICE for time to first recurrence. However, when maintenance was given, TICE was more effective than Connaught for time to first recurrence with a positive trend for time to death due to bladder cancer. For time to progression and OS, Connaught

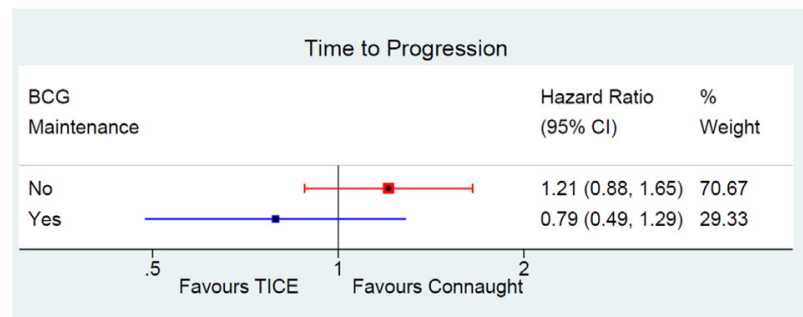

Fig. 2. Forest plot time to progression. (Color version of figure is available online.)

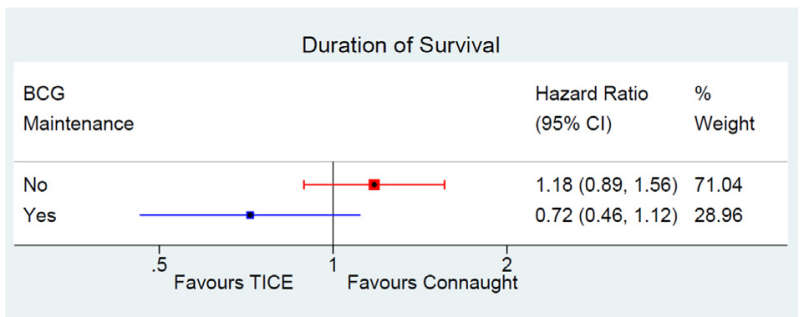

Fig. 3. Forest plot duration of survival. (Color version of figure is available online.)

and TICE had similar efficacy. Compared to no maintenance therapy, maintenance BCG significantly reduced the risk of recurrence, progression, and death for TICE, but not for Connaught.

Why is Connaught better when no maintenance is given whereas TICE seems to be better with longer treatment? Mice experiments from Rentsch showed a stronger immune response to Connaught than to TICE BCG [2]. This might explain the initial advantage of Connaught. However, we also know that immunotherapy, in contrast to chemotherapy, is more likely to have an optimal biologically effective dose than a maximal effective dose. This, for example, was shown for intravesical BCG by de Reijke et al. [6]. Looking at urinary interleukin (IL)-2 kinetics, IL2 tended to be higher during the first course and lower during the second and third course of BCG, suggesting a decreasing immune response with time. Combining these findings, one might hypothesize that Connaught reaches an earlier cumulative optimal dose and clinical effect as compared with TICE because of a stronger immune response. On the contrary, Connaught might lose some of its efficacy during maintenance therapy when TICE has reached its optimum.

Without maintenance, our lower risk of recurrence on Connaught is in line with the study by Rentsch et al. [2] who reported a significantly higher 5-year RFS rate, 74\% (62.8\%-87.2\%) for Connaught compared to $48 \%$ (35.5\%$65.1 \%)$ for TICE, $P=0.01$. Their 5 -year progression rates were $5.9 \%$ for Connaught and $12.9 \%$ for TICE $(P=0.34)$. In a multivariate analysis, BCG strain remained the only significant variable for recurrence (HR 2.91). Their mice experiments showed less TICE bacilli in lymph nodes and a stronger immune response to Connaught in similar numbers

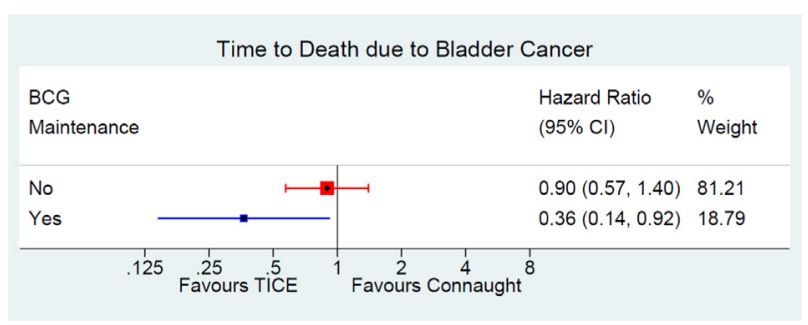

Fig. 4. Forest plot time to death due to bladder cancer. (Color version of figure is available online.) 
of live bacilli in both strains. It is noteworthy that the increased immune response in the bladder did not cause more local bladder symptoms. Connaught patients had even less dysuria, $13 \%$, vs. $30 \%$ with TICE, $P=0.015$. Limitations of their study are the low patient numbers (142 vs. a goal of 300) and the long recruitment period (12 y).

Only a few studies have previously studied different BCG strains. Kaisary [7] compared BCG Glaxo and BCG Pasteur, initiated because results with BCG in Great Britain were disappointing. In this pilot study with 21 patients, results between the strains were similar.

In a 1994 Medical Research Council randomized marker lesion study, Fellows et al. [8] compared BCG Pasteur and Evans BCG. In 99 patients, all papillary tumors, but one, were resected and a course of 6 BCG instillations was given. In 51 eligible patients on Evans BCG, 27 (53\%) still had a marker lesion or lesions at other sites at the 3 months evaluation compared with 16 (37\%) of 46 eligible patients in the Pasteur group. The difference was only "statistically suggestive."

In 1995, a Dutch study compared 6 weeks of BCG TICE, 6 weeks of BCG RIVM, and 6 months of mitomycin$\mathrm{C}$ in 437 patients with NMIBC [3]. The 5-year recurrencefree rates with BCG TICE were the lowest $(36 \pm 5 \%)$ and significantly less than with mitomycin-C $(57 \pm 5 \%), P=$ 0.01 . The 5-year recurrence-free rates with BCG RIVM were also better $(54 \pm 5 \%)$ than those with TICE BCG, but the difference was not statistically significant. So again, results with BCG TICE were suboptimal without maintenance.

A recent study compared a 6 to 8 week course of BCG Tokyo172 and BCG Connaught [4]. The study was stopped in 2012, because Connaught production was suspended. From 2004 until 2012, 178 patients were enrolled, of which 66 Tokyo172 and 63 Connaught patients were analyzed. After a median follow-up of almost 2.5 years, the complete response rates of concomitant patients with CIS were $90.3 \%$ and $85.0 \%$ for Tokyo172 and Connaught, respectively $(P=0.896)$. The 2-year RFS rates were not significantly different: $73.2 \%$ and $68.8 \%$, respectively. These 2 strains have also been compared in a mouse model [9]. Tumor cells with various doses of both BCG strains were injected in the flank of syngenic mice.Tokyo172 achieved similar tumor suppression at a lower dose than Connaught. However, although Tokyo172 had approximately half the bacilli compared with Connaught, the colony-forming unit content was approximately 13-fold higher for Tokyo172. Tokyo172 bacilli were also better dispersed after reconstitution and Tokyo172 bacilli showed better attachment to tumor cells in vitro. The authors concluded that these differences in favor of Tokyo172 were the main reasons for a lower dose of Tokyo172 to be clinically effective (18 vs. $81 \mathrm{mg}$ dry weight).

Other nonclinical studies have addressed potential differences between different BCG strains. Secanella et al. [10] used 3 evolutionary early and 5 late BCG substrains, with genetic and antigenic differences, in bladder cancer cell lines. As in the use of BCG vaccines, antitumor effect and IL-6 and IL-8 response differed between BCG strains and cell line used. For T24 and J82 cells, for example, Russian and Connaught BCG strains (an evolutionary early and late substrain) were more effective in cell proliferation inhibition and cytokine response as compared with 6 other BCG strains, including TICE BCG.

It is conceivable that serial passages have resulted in genetic mutations and alterations, which have resulted in differences in phenotype, antigenicity, and subsequent clinical efficacy and toxicity of the different substrains that are currently used for intravesical treatment.

The strength of this study is that it is a large homogeneous group of patients with $\mathrm{T} 1 \mathrm{G} 3$ previously untreated with BCG. A limitation is that it is a retrospective study in a selected patient group without central pathology review. A subset of patients (41\%) had a second resection; however, adjustment for a reTUR did not change the overall conclusions. Neither the BCG strain nor the decision to give maintenance treatment were randomized. Finally, BCG maintenance schedules and their duration were not standardized. However, approximately one-third of patients receiving maintenance were treated with the SWOG 3-year maintenance schedule in both strains. A prospective randomized trial with sufficient patients and adequate and clearly defined treatment schedules is needed to confirm these results.

\section{Conclusion}

We report on the difference in efficacy between the 2 most commonly used BCG strains. In a large retrospective dataset of T1 high-grade patients previously untreated with BCG, our results show that without maintenance, Connaught was more effective than TICE for the time to first recurrence potentially because of a stronger initial immune response caused by Connaught BCG. However, when maintenance was given, TICE was more effective than Connaught for the time to first recurrence.

\section{References}

[1] Kamat AM, Witjes JA, Brausi M, Soloway M, Lamm D, Persad R, et al. Defining and treating the spectrum of intermediate-risk NMIBC. J Urol 2014;192:305-15.

[2] Rentsch CA, Birkhäuser FD, Biot C, Gsponer JR, Bisiaux A, Wetterauer C, et al. Bacillus Calmette-Guérin strain differences have an impact on clinical outcome in bladder cancer immunotherapy. Eur Urol 2014;66: 677-88.

[3] Vegt PDJ, Witjes JA, Witjes WPJ, Doesburg WH, Debruyne FMJ, van der Meijden APM. A randomized study of intrasvesical mitomycin-C, bacillus Calmette-Guerin Tice and bacillus Calmette-Guerin RIVM in pTa-pT1 papillary carcinoma and carcinoma in situ of the bladder. J Urol 1995;153:929-33.

[4] Sengiku A, Ito M, Miyazaki Y, et al. A prospective comparative study of intravesical bacillus Calmette-Guerin therapy with the Tokyo or 
Connaught strain for nonmuscle invasive bladder cancer. J Urol 2013;190:50-4.

[5] Gontero P, Sylvester R, Pisano F, Joniau S, Vander Eeckt K, Serretta $\mathrm{V}$, et al. Prognostic factors and risk groups in T1G3 non-muscleinvasive bladder cancer patients initially treated with bacillus Calmette-Guérin: results of a retrospective multicenter study of 2451 patients. Eur Urol 2015;67:74-82.

[6] de Reijke TM, deBoer EC, Kurth KH, Schamhart DHJ Urinary interleukin-2 monitoring during prolonged BCG treatment: can it predict the optimal number of instillations? J Urol 1999;161:67-71.

[7] Kaisary AV. Intravesical BCG therapy in the management of multiple superficial bladder carcinoma. Comparison between Glaxo and Pasteur strains. Br J Urol 1987;59:554-8.
[8] Fellows GJ, Parmar MK, Grigor KM, Hall RR, Heal MR, Wallace DM. Marker tumour response to Evans and Pasteur bacille Calmette-Guérin in multiple recurrent $\mathrm{pTa} / \mathrm{pT} 1$ bladder tumours: report from the Medical Research Council Subgroup on Superficial Bladder Cancer (Urological Cancer Working Party). Br J Urol 1994;73:639-44.

[9] Ikeda N, Honda I, Yano I, Koyama A, Toida I. Bacillus CalmetteGuerin Tokyo172 substrain for superficial bladder cancer: characterization and antitumor effect. J Urol 2005;173:1507-12.

[10] Secanella Fandos S, Luquin M, Julian E. Connaught and Russian strains showed the highest direct antitumor effects of different bacillus Calmette-Guérin substrains. J Urol 2013;189:711-8. 